\title{
Diagnostic Imaging to Exclude Acute Coronary Syndrome
}

\author{
Simon A. Mahler $\cdot$ Chadwick D. Miller
}

Published online: 22 December 2012

(c) Springer Science+Business Media New York 2012

\begin{abstract}
On virtually every shift, Emergency Department providers care for patients with chest pain. With each encounter, the provider has the difficult task of determining if the patient's pain is of cardiac or non-cardiac origin. This is a high-stakes decision, as patients discharged with an incorrect diagnosis of non-cardiac chest pain are at high risk for morbidity and mortality, while the provider is at high risk for a medical malpractice claim (Pope et al., N Engl J Med 342(16):1163-1170, 2000; Amsterdam et al., Circulation 122(17):1756-1776, 2010). Diagnostic imaging studies, such as coronary computed tomography angiography, cardiac magnetic resonance imaging, stress echocardiography, and nuclear imaging, play an important role in the risk stratification of patients presenting with chest pain or other symptoms concerning for acute coronary syndrome (ACS). This article will evaluate and summarize the recent evidence relevant to diagnostic imaging modalities used to exclude ACS.
\end{abstract}

Keywords Coronary computed tomography angiography · Cardiac magnetic resonance imaging . Stress echocardiography · Nuclear perfusion imaging . Acute coronary syndrome

\footnotetext{
S. A. Mahler

Department of Epidemiology and Prevention, Wake Forest School of Medicine, Medical Center Boulevard, Winston Salem, NC 27157, USA

\section{S. A. Mahler $(\varangle) \cdot$ C. D. Miller}

Department of Emergency Medicine, Wake Forest School of Medicine, Medical Center Boulevard, Winston Salem, NC 27157, USA

e-mail: smahler@wakehealth.edu
}

\section{Introduction}

Approximately $8-10$ million patients complaining of chest pain present to an Emergency Department (ED) annually in the United States [1]. Although chest pain is a frequent complaint, chest pain risk stratification remains challenging. In the US, 50 to $70 \%$ of ED patients with chest pain are admitted, yet only about $10 \%$ are ultimately diagnosed with acute coronary syndrome (ACS) [2-5]. In addition, chest pain is a significant contributor to US health care costs, estimated at \$5-10 billion annually [6]. Despite high admission rates and costs, $2-5 \%$ of patients with AMIs are missed on initial presentation and discharged from the ED [7]. Multiple imaging strategies with high negative predictive values have been developed to more accurately risk-stratify patients with symptoms concerning for ACS. In addition, some of these imaging strategies have been reported to decrease patient length of stay, speed diagnosis, and limit downstream utilization and costs.

Rationale for Imaging

The initial approach to a patient with symptoms concerning for ACS includes risk stratification based on a history, physical examination, and ancillary testing such as ECGs and cardiac biomarkers. Studies have demonstrated that certain historical factors increase or decrease the likelihood for ACS. Radiation to the arms and shoulders, exertional chest pain, and associated symptoms of dyspnea, diaphoresis, nausea or vomiting are associated with an increased likelihood of ACS [8-10]. Sharp, pleuritic, positional chest pain is associated with a decreased likelihood of ACS [10]. However, there are no historical features with sufficient sensitivity and specificity to rule-in or -out the diagnosis of ACS. Furthermore, the physical examination of patients 
with ACS is often normal and no examination findings are sufficiently sensitive or specific to rule-in or exclude ACS.

Major risk factors for coronary artery disease identified by large epidemiologic studies include age $>40$ years old, male or postmenopausal female, hypertension, cigarette smoking, hypercholesterolemia, diabetes, truncal obesity, family history, and sedentary lifestyle [11, 12].

While cardiac risk factors are useful in predicting coronary artery disease and ACS-risk within a given population, they are less useful for diagnosing the presence or absence of ACS in an individual patient $[10,13,14]$. Male sex, hypertension, diabetes, tobacco use, elevated cholesterol, and a positive family history all have LRs close to 1.0 , indicating no diagnostic value for prediction of ACS [10].

Ancillary testing in patients with possible ACS typically includes electrocardiography (ECG) and cardiac biomarkers. However, like history and physical examination, even normal ECGs and cardiac biomarkers do not exclude ACS. While a normal ECG is reassuring (LR of $0.2,95 \% \mathrm{CI}$ 0.1-0.3 for AMI), when it is used alone it lacks the sensitivity to reliably exclude ACS. In a large multicenter observational study of 391,208 patients with an evaluable ECG and diagnosis of AMI, $63 \%$ had "diagnostic" ECG changes, $39 \%$ had non-specific changes, and $8 \%$ had normal ECGs. Diagnostic changes were defined as ST-segment elevation, ST-segment depression, or left bundlebranch block [15]. Other studies have documented normal or near-normal ECGs in 5-10\% of patients with AMI [16-19]. A normal ECG is also an independent risk factor for missed AMI and inappropriate ED discharge (odds ratio 7.7, 95 \%CI 2.9-20.2) [2]. Guidelines recommend measurement of a cardiac troponin assay in all patients with suspected ACS [7]. However, contemporary troponin assays will not identify all patients with ACS, as patients with unstable angina, who are at high risk for near-term AMI, typically have normal troponins.

Given the limitations of the history, physical, examination, ECGs, and laboratory tests in excluding ACS, the American College of Cardiology/American Heart Association (ACC/AHA) guidelines suggest that patients with possible ACS (including low-risk patients) should receive stress testing or advanced cardiac imaging [20]. Imaging modalities such as coronary computed tomography angiography (CCTA) and cardiac magnetic resonance imaging (CMR) are gaining popularity at academic and large community hospitals, while nuclear imaging and stress echocardiography remain mainstays at many hospitals. Recent advances in stress testing and cardiac imaging modalities used to exclude ACS are discussed below.

Although history, physical examination, and ancillary testing lack sensitivity to exclude ACS, they remain an essential component of every patient's evaluation. Diagnostic imaging should not be used as a replacement for a thorough history and physical examination. History and examination findings remain vital to the identification or exclusion of other causes of chest pain and to select the most appropriate imaging modality. Patients should also be queried for prior events and prior diagnostic testing, and the patient's presenting symptoms should be interpreted in light of those results.

\section{Coronary Computed Tomography Angiography}

CCTA has emerged as an important tool for risk-stratifying patients presenting to the ED with chest pain. Unlike stress tests which provide functional assessments for myocardial ischemia, CCTA provides anatomic information regarding the patency of coronary arteries. Findings of coronary stenosis on CCTA closely correlate with findings on invasive coronary angiography [21]. The sensitivity and negative predictive value of CCTA for coronary artery disease (CAD) are high, approximately $95 \%$ and $99 \%$, respectively [22]. Several studies have demonstrated that the negative predictive value for ACS remains high for up to 2 years [23-27, 28•, 29•]. Additionally, annualized adverse cardiac event rates after a normal CCTA (i.e., no coronary disease) are exceedingly low (0.17\%) [30] and similar to the event rates after a negative stress echo $(0.54 \%)$ and stress nuclear $(0.45 \%)$ imaging [31]. Therefore ED or Observation Unit (OU) patients with no evidence of coronary stenosis on CCTA can be discharged without further testing, and are unlikely to require additional diagnostic imaging for at least 2 years.

Unlike stress modalities which require at least two negative troponins be completed prior to imaging, CCTA can be completed in parallel with serial biomarker assessment [28•, 29•]. This provides CCTA with a potential efficiency advantage over stress testing modalities which may enhance ED or OU through-put. Two recent large multicenter randomized clinical trials have demonstrated that hospital length of stay is shorter among ED patients imaged with CCTA compared to other stress testing and imaging modalities [28•, 29•]. In addition, CCTA is associated with a decreased time-to-diagnosis and an increase in the number of patients that can be discharged directly from the ED [28•, 29•]. However, studies demonstrating enhanced efficiency from using CCTA have not adequately explored the relationship between CCTA availability and improved efficiency. CCTA is unlikely to improve length of stay when it is ordered in patients presenting during hours in which CCTA is not immediately available [32].

Care should be taken to select patients for CCTA, as up to $25 \%$ of patients presenting with symptoms concerning for ACS may have relative or absolute contraindications [33]. CCTA should be avoided in patients with renal dysfunction and allergies to iodinated contrast agents. Patients 
with tachycardia, obesity, advanced age, known coronary artery disease (CAD), or multiple risk factors for CAD are also poor candidates for CCTA, as these findings are associated with an increased rate of non-diagnostic CCTAs due to poor imaging quality $[34,35]$.

CCTA appears to have more diagnostic utility in patients at low to intermediate risk for ACS rather than those at high risk. CCTA accurately determines if coronary disease is present, but does not provide any functional assessment of the heart. Therefore, routine CCTA cannot differentiate ischemic from non-ischemic chest pain in patients with known coronary artery disease. In the highrisk patients, especially those with known CAD, other diagnostic imaging modalities such as CMR or nuclear imaging may be preferable to CCTA.

Potential drawbacks to the use of CCTA for ED ACS risk stratification include radiation exposure, costs, and increased downstream test utilization. While the mean radiation exposure for 64-slice CCTA in the ROMICAT II clinical trial was $12.3 \mathrm{mSv}$ [29॰], advances in technology now permit CCTA at much lower radiation doses, which will mitigate these risks.

The cost of CCTA has been shown to be similar to other stress-testing modalities [29•]. However, CCTA is associated with increasing downstream testing, including functional stress-testing and invasive coronary imaging in some patient populations [29•, 36]. However, this could be offset by avoiding imaging during return visits in patients with negative CCTA imaging. Future analyses will need to elucidate this intermediate-term effect on resource utilization. In addition, CCTA requires a highly trained technical staff and specialized radiologists for interpretation, which often leads to a lack of availability for CCTA during weekends and off-hours.

\section{Cardiac Magnetic Resonance Imaging}

The use of stress cardiac magnetic resonance (CMR) imaging to evaluate ED patients with chest pain is a relatively recent adaption of this technology. A major advantage of CMR is the ability to acquire a comprehensive assessment of cardiac structure and function during a single examination. This imaging technique allows for the distinction between new and old infarctions, detection of edema (often an early marker of ischemia), and evaluation for inducible ischemia. Downsides of CMR include patient intolerance due to claustrophobia, contraindications associated with metallic objects (e.g., pacemakers), and the time required for image acquisition.

Relative to other technologies, CMR appears to have the highest accuracy. Recently the "cardiovascular magnetic resonance and single-photon emission computed tomography for diagnosis of coronary heart disease" (CE-MARC) trial compared CMR to single-photon emission computed tomography (SPECT) imaging in 752 patients [37]. CMR was more sensitive ( $87 \%$ vs $67 \%, p<0.001$ ) for the detection of significant coronary disease, with both modalities demonstrating $84 \%$ specificity. The findings in this trial are consistent with previous investigations comparing CMR to SPECT. Similarly, CMR has also been shown to be more sensitive and specific than stress echocardiography [38].

In ED patients, resting CMR has been shown to be highly sensitive for early signs of ischemia, even detecting infarction before elevated serum troponin elevation occurs [39]. After exclusion of MI with serial troponin measurements, vasodilator stress CMR in ED patients was $100 \%$ sensitive for the manifestation of significant CAD at 1 year in 135 subjects [40]. Most recently, the ability to adapt stress CMR imaging to patients in an ED observation unit (OU) was evaluated. In one trial, a strategy of OU care with stress CMR imaging in intermediate- to high-risk patients reduced cost during the index visit and through 1 year compared to inpatient care without any detectable differences in clinical outcomes [41•]. A second trial in lowerrisk patients compared two OU imaging approaches: a mandatory CMR strategy versus a provider choice strategy [42•]. Among these strategies, no differences were detected in length of stay, appropriateness of admission decisions, or the performance of invasive angiography without revascularization. However, in these lower-risk patients, the OU-CMR strategy was associated with an increase in cost compared to the provider choice strategy (median cost $\$ 2005$ versus $\$ 1686, p<0.001$ ). Overall, these two trials suggest the most beneficial application of CMR is in higher-risk, more complex patients, especially when used in an $\mathrm{OU}$ as an alternative strategy to inpatient admission.

\section{Stress Echocardiography}

Echocardiography is a commonly used diagnostic imaging modality for ACS risk stratification. Exercise or pharmacologic stress echocardiograms detect regional wall motion abnormalities induced by myocardial ischemia. Wall motion abnormalities are often detectable almost immediately after the onset of ischemia, which may precede classic findings such as ECG changes [43]. Positive stress echocardiography is strongly predictive of CAD ( $\mathrm{LR}=8.6$ ) [44]. A recent metaanalysis estimated that patients with negative exercise stress echocardiography had an annualized rate of myocardial infarction or cardiac death of $0.54 \%$ per year, which was comparable to nuclear imaging [31]. However, patients with negative stress echocardiography had a slightly lower rate of revascularization and unstable angina over 1 year $(0.95 \%)$ compared to nuclear imaging (1.25\%) [31]. 
Myocardial contrast stress echocardiography involves the addition of contrast agents to quantify myocardial profusion in addition to improving the detection of wall motion defects. Use of contrast echocardiography has been shown to increase the sensitivity for detection of CAD relative to non-contrast echocardiography [45-47]. Patients with normal perfusion and normal wall motion on contrast stress echocardiography were found to have a $98 \%$ negative predictive value for cardiac events at 2 years [47].

Advantages of echocardiography relative to other modalities include availability, portability, and a lack of ionizing radiation. However, in order for a wall motion abnormality to be detected greater than $20 \%$ of the transmural thickness of the myocardium must be affected [48]. Furthermore, failure of the patient to achieve a heart rate at or above $85 \%$ of target may result in a stress that is inadequate to precipitate a wall motion abnormality. Other limitations include difficulty distinguishing new versus old wall motion abnormalities, and lower accuracy in some subgroups. For instance, some studies have suggested that echocardiography may be less accurate in women than in men [49]. Additionally, stress echo has a sensitivity of $36 \%$ for significant coronary stenosis in those with concentric left ventricular hypertrophy, and a sensitivity of $56 \%$ for left circumflex disease [50, 51]. In aggregate, these characteristics make stress echo most adaptable to lowand intermediate-risk, less complicated patients.

\section{Nuclear Stress Imaging}

Single-photon emission computed tomography (SPECT) imaging has been a mainstay of cardiac imaging for the past several decades. With SPECT, a radioactive tracer is injected during stress and can be compared to the distribution during rest to detect the presence of inducible ischemia. Similar to CCTA, this modality involves patient exposure to ionizing radiation. In the ED setting, SPECT has proven ability to accurately detect ongoing ischemia and/or inducible ischemia. Large observational cohorts involving thousands of patients have demonstrated the high sensitivity of SPECT for clinical events in ED and OU patients with acute chest pain $[52,53]$. The rate of adverse cardiac events after a negative stress SPECT exam is $0.45 \%$, similar to stress echo [31].

Recent investigations have compared CMR to SPECT. In the CE-MARK trial (discussed above), CMR was shown to have higher sensitivity than SPECT, but no differences in specificity were detected [37]. In the MR-IMPACT II study, among 515 patients, CMR had higher sensitivity (67\% vs $59 \%$ ) but lower specificity (61\% vs $72 \%$ ) for significant coronary stenosis ( $>50 \%$ ) compared to stress SPECT [54]. Notably, neither of these trials investigated ED patients with acute symptoms.
The availability, proven track record, and accuracy of stress/rest SPECT make this modality a trusted option for imaging patients with acute chest pain. The radiation exposure associated with this modality is the primary drawback. Similar to CMR, this modality appears most useful in non-low-risk patients most likely to benefit from robust perfusion imaging.

\section{Conclusions}

Providers have four primary choices for diagnostic imaging modalities when evaluating patients with acute chest pain. Selection among these modalities is often determined by institutional strengths and availability. Two choices, stress echo and stress SPECT, are widely available, accurate, and have longstanding, proven accuracy. Of these two modalities, stress echo is most adaptable to lower-risk patients, whereas the strength of perfusion imaging with SPECT is most useful in more complex patients. Two new options include CCTA and CMR. CCTA has demonstrated utility in excluding CAD among low-risk patients. Similar to SPECT, the strengths of CMR imaging make it most useful in more complex, higher-risk patients. With all of these modalities, good quality, negative examinations are associated with low rates of adverse cardiac events at 1 year.

Acknowledgments Dr. Mahler receives research funding from the American Heart Association (12CPR12000001). Dr. Miller: This publication was made possible by Grant Number R21 HL09713101A1 from NHLBI. Its contents are solely the responsibility of the authors and do not necessarily represent the official views of NHLBI.

Disclosure C. Miller: Research support from Siemens, Alere Scarborough, Inc, Dyax Corp, 3 M, Pennsylvania Department of Health; consultant for Mylan Specialty LP; medical malpractice case review; S. Mahler: nothing to disclose (current and past 12 months).

\section{References}

Papers of particular interest, published recently, have been highlighted as:

- Of importance

1. Owens PL, Barrett ML, Gibson TB, Andrews RM, Weinick RM, Mutter RL. Emergency department care in the United States: a profile of national data sources. Ann Emerg Med. 2010; 56(2):150-65.

2. Pope JH, Aufderheide TP, Ruthazer R, et al. Missed diagnoses of acute cardiac ischemia in the emergency department. N Engl $\mathrm{J}$ Med. 2000;342(16):1163-70.

3. Pines JM, Isserman JA, Szyld D, Dean AJ, McCusker CM, Hollander JE. The effect of physician risk tolerance and the presence of an observation unit on decision making for ED patients with chest pain. Am J Emerg Med. 2010;28(7):771-9. 
4. Fleischmann KE, Goldman L, Johnson PA, et al. Critical pathways for patients with acute chest pain at low risk. J Thromb Thrombolysis. 2002;13(2):89-96.

5. Roger VL, Go AS, Lloyd-Jones DM, et al. Heart disease and stroke statistics-2011 update: a report from the American Heart Association. Circulation. 2011;123(4):e18-209.

6. Gomez MA, Anderson JL, Karagounis LA, Muhlestein JB, Mooers FB. An emergency department-based protocol for rapidly ruling out myocardial ischemia reduces hospital time and expense: results of a randomized study (ROMIO). J Am Coll Cardiol. 1996;28(1):25-33.

7. Amsterdam EA, Kirk JD, Bluemke DA, et al. Testing of low-risk patients presenting to the emergency department with chest pain: a scientific statement from the American Heart Association. Circulation. 2010;122(17):1756-76.

8. Panju AA, Hemmelgarn BR, Guyatt GH, Simel DL. The rational clinical examination. Is this patient having a myocardial infarction? JAMA. 1998;280(14):1256-63.

9. Goodacre S, Locker T, Morris F, Campbell S. How useful are clinical features in the diagnosis of acute, undifferentiated chest pain? Acad Emerg Med. 2002;9(3):203-8.

10. Chun AA, McGee SR. Bedside diagnosis of coronary artery disease: a systematic review. Am J Med. 2004;117(5):334-43.

11. Stokes J 3rd, Kannel WB, Wolf PA, Cupples LA, D’Agostino $\mathrm{RB}$. The relative importance of selected risk factors for various manifestations of cardiovascular disease among men and women from 35 to 64 years old: 30 years of follow-up in the Framingham Study. Circulation. 1987;75(6 Pt 2):V65-73.

12. Sytkowski PA, Kannel WB, D'Agostino RB. Changes in risk factors and the decline in mortality from cardiovascular disease. The Framingham Heart Study. N Engl J Med. 1990;322(23):1635-41.

13. Swap CJ, Nagurney JT. Value and limitations of chest pain history in the evaluation of patients with suspected acute coronary syndromes. JAMA. 2005;294(20):2623-9.

14. Han JH, Lindsell CJ, Storrow AB, et al. The role of cardiac risk factor burden in diagnosing acute coronary syndromes in the emergency department setting. Ann Emerg Med. Feb 2007;49(2):145-52, 152 e141.

15. Welch RD, Zalenski RJ, Frederick PD, et al. Prognostic value of a normal or nonspecific initial electrocardiogram in acute myocardial infarction. JAMA. 2001;286(16):1977-84.

16. Slater DK, Hlatky MA, Mark DB, Harrell FE Jr, Pryor DB, Califf RM. Outcome in suspected acute myocardial infarction with normal or minimally abnormal admission electrocardiographic findings. Am J Cardiol. 1987;60(10):766-70.

17. Kontos MC, Desai PV, Jesse RL, Ornato JP. Usefulness of the admission electrocardiogram for identifying the infarct-related artery in inferior wall acute myocardial infarction. Am J Cardiol. 1997;79(2):182-4.

18. Forest RS, Shofer FS, Sease KL, Hollander JE. Assessment of the standardized reporting guidelines ECG classification system: the presenting ECG predicts 30-day outcomes. Ann Emerg Med. 2004;44(3):206-12.

19. Kontos MC, Diercks DB, Kirk JD. Emergency department and office-based evaluation of patients with chest pain. Mayo Clin Proc. 2010;85(3):284-99.

20. Anderson JL, Adams CD, Antman EM, et al. ACC/AHA 2007 guidelines for the management of patients with unstable angina/ non-ST-Elevation myocardial infarction: a report of the American College of Cardiology/American Heart Association Task Force on Practice Guidelines (Writing Committee to Revise the 2002 Guidelines for the Management of Patients With Unstable Angina/Non-ST-Elevation Myocardial Infarction) developed in collaboration with the American College of Emergency Physicians, the Society for Cardiovascular Angiography and Interventions, and the Society of Thoracic Surgeons endorsed by the
American Association of Cardiovascular and Pulmonary Rehabilitation and the Society for Academic Emergency Medicine. J Am Coll Cardiol. 2007;50(7):e1-157.

21. Andreini D, Pontone G, Ballerini G, et al. Feasibility and diagnostic accuracy of 16-slice multidetector computed tomography coronary angiography in 500 consecutive patients: critical role of heart rate. Int J Cardiovasc Imaging. 2007;23(6):789-801.

22. Budoff MJ, Dowe D, Jollis JG, et al. Diagnostic performance of 64-multidetector row coronary computed tomographic angiography for evaluation of coronary artery stenosis in individuals without known coronary artery disease: results from the prospective multicenter ACCURACY (Assessment by Coronary Computed Tomographic Angiography of Individuals Undergoing Invasive Coronary Angiography) trial. J Am Coll Cardiol. 2008;52(21):1724-32.

23. Aldrovandi A, Maffei E, Palumbo A, et al. Prognostic value of computed tomography coronary angiography in patients with suspected coronary artery disease: a 24-month follow-up study. Eur Radiol. 2009;19(7):1653-60.

24. Hoffmann U, Bamberg F, Chae CU, et al. Coronary computed tomography angiography for early triage of patients with acute chest pain: The ROMICAT (Rule Out Myocardial Infarction using Computer Assisted Tomography) Trial. J Am Coll Cardiol. 2009;53(18):1642-50.

25. Hollander JE, Chang AM, Shofer FS, et al. One-year outcomes following coronary computerized tomographic angiography for evaluation of emergency department patients with potential acute coronary syndrome. Acad Emerg Med. 2009;16(8):693-8.

26. Goldstein JA, Gallagher MJ, O’Neill WW, Ross MA, O’Neil BJ, Raff GL. A randomized controlled trial of multi-slice coronary computed tomography for evaluation of acute chest pain. J Am Coll Cardiol. 2007;49(8):863-71.

27. Schlett CL, Banerji D, Siegel E, et al. Prognostic value of CT angiography for major adverse cardiac events in patients with acute chest pain from the emergency department: 2-year outcomes of the ROMICAT trial. JACC Cardiovasc Imaging. 2011;4(5):481-91.

28. - Litt HI, Gatsonis C, Snyder B, et al. CT angiography for safe discharge of patients with possible acute coronary syndromes. N Engl J Med. 2012;366(15):1393-403. Demonstrates that a CCTA-based risk stratification strategy for low- to intermediaterisk patients safely decreases Emergency Department length of stay compared to patients receiving stress testing strategy

29. • Hoffmann U, Truong QA, Schoenfeld DA, et al. Coronary CT angiography versus standard evaluation in acute chest pain. N Engl J Med. 2012;367(4):299-308. Demonstrates that CCTA safely decreases Emergency Department length of stay compared to patients receiving stress testing without significantly increasing costs. However CCTA is associated with increased downstream testing and radiation exposure

30. Hulten EA, Carbonaro S, Petrillo SP, Mitchell JD, Villines TC. Prognostic value of cardiac computed tomography angiography: a systematic review and meta-analysis. J Am Coll Cardiol. 2011;57(10):1237-47.

31. Metz LD, Beattie M, Hom R, Redberg RF, Grady D, Fleischmann KE. The prognostic value of normal exercise myocardial perfusion imaging and exercise echocardiography: a meta-analysis. J Am Coll Cardiol. 2007;49(2):227-37.

32. Mahler SA. Length of stay for observation unit chest pain patients tested with coronary computed tomography angiography compared to stress testing depends on time of emergency department presentation. Acad Emerg Med. 2012;April:19(S1).

33. Goldstein JA, Gallagher MJ, O’Neill WW, Ross MA, O’Neil BJ, Raff GL. A randomized controlled trial of multi-slice coronary computed tomography for evaluation of acute chest pain. J Am Coll Cardiol. 2007;49(8):863-71. 
34. Bamberg F, Abbara S, Schlett CL, et al. Predictors of image quality of coronary computed tomography in the acute care setting of patients with chest pain. Eur J Radiol. 2010;74(1):182-8.

35. Dewey M, Vavere AL, Arbab-Zadeh A, et al. Patient characteristics as predictors of image quality and diagnostic accuracy of MDCT compared with conventional coronary angiography for detecting coronary artery stenoses: CORE-64 Multicenter International Trial. AJR Am J Roentgenol. 2010;194(1):93-102.

36. Shreibati JB, Baker LC, Hlatky MA. Association of coronary CT angiography or stress testing with subsequent utilization and spending among Medicare beneficiaries. JAMA. 2011;306(19):2128-36.

37. Greenwood JP, Maredia N, Younger JF, et al. Cardiovascular magnetic resonance and single-photon emission computed tomography for diagnosis of coronary heart disease (CE-MARC): a prospective trial. Lancet. 2012;379(9814):453-60.

38. Nagel E, Lehmkuhl HB, Bocksch W, et al. Noninvasive diagnosis of ischemia-induced wall motion abnormalities with the use of highdose dobutamine stress MRI. Circulation. 1999;99(6):763-70.

39. Cury RC, Shash K, Nagurney JT, et al. Cardiac magnetic resonance with T2-weighted imaging improves detection of patients with acute coronary syndrome in the emergency department. Circulation. 2008;118(8):837-44.

40. Ingkanisorn WP, Kwong RY, Bohme NS, et al. Prognosis of negative adenosine stress magnetic resonance in patients presenting to an emergency department with chest pain. J Am Coll Cardiol. 2006;47(7):1427-32.

41. - Miller CD, Hwang W, Case D, et al. Stress CMR imaging observation unit in the emergency department reduces 1-year medical care costs in patients with acute chest pain: a randomized study for comparison with inpatient care. JACC: Cardiovasc Imaging. 2011;4(8):862-70. Demonstrates that an Observation Unit based CMR strategy for the risk stratification of intermediate- to high-risk patients safely reduces cost during the index visit and through 1 year compared to inpatient care.

42. - Miller CD, Hoekstra JW, Lefebvre C, et al. Provider-directed imaging stress testing reduces health care expenditures in lower-risk chest pain patients presenting to the emergency department/clinical perspective. Circul Cardiovasc Imaging. 2012;5(1):111-8. Demonstrates that an Observation Unit based CMR strategy for the risk stratification of Low-risk patients increases costs compared to provider-directed stress testing. These findings in combination with those from reference 41 suggest that CMR has more utility in patients at intermediate- to high-risk for ACS rather than those at low risk.

43. Hauser AM, Gangadharan V, Ramos RG, Gordon S, Timmis GC. Sequence of mechanical, electrocardiographic and clinical effects of repeated coronary artery occlusion in human beings: echocardiographic observations during coronary angioplasty. J Am Coll Cardiol. 1985;5(2 Pt 1):193-7.

44. Banerjee A, Newman DR, Van den Bruel A, Heneghan C. Diagnostic accuracy of exercise stress testing for coronary artery disease: a systematic review and meta-analysis of prospective studies. Int J Clin Pract. 2012;66(5):477-92.

45. Senior R, Shah BN. Myocardial contrast echocardiography for simultaneous assessment of function and perfusion in real time: a technique comes of age. 2012.

46. Plana JC, Mikati IA, Dokainish H, et al. A randomized cross-over study for evaluation of the effect of image optimization with contrast on the diagnostic accuracy of dobutamine echocardiography in coronary artery disease The OPTIMIZE Trial. JACC Cardiovasc Imaging. 2008;1(2):145-52.

47. Gaibazzi N, Reverberi C, Lorenzoni V, Molinaro S, Porter TR. Prognostic value of high-dose dipyridamole stress myocardial contrast perfusion echocardiography. Circulation. 2012.

48. Lieberman AN, Weiss JL, Jugdutt BI, et al. Two-dimensional echocardiography and infarct size: relationship of regional wall motion and thickening to the extent of myocardial infarction in the dog. Circulation. 1981;63(4):739-46.

49. Kwok Y, Kim C, Grady D, Segal M, Redberg R. Meta-analysis of exercise testing to detect coronary artery disease in women. Am J Cardiol. 1999;83(5):660-6.

50. Smart SC, Knickelbine T, Malik F, Sagar KB. Dobutamineatropine stress echocardiography for the detection of coronary artery disease in patients with left ventricular hypertrophy. Importance of chamber size and systolic wall stress. Circulation. 2000;101(3):258-63.

51. Geleijnse ML, Fioretti PM, Roelandt JRTC. Methodology, feasibility, safety and diagnostic accuracy of dobutamine stress echocardiography. J Am Coll Cardiol. 1997;30(3):595-606.

52. Tatum JL, Jesse RL, Kontos MC, et al. Comprehensive strategy for the evaluation and triage of the chest pain patient. Ann Emerg Med. 1997;29(1):116-25.

53. Fesmire FM, Hughes AD, Stout PK, Wojcik JF, Wharton DR. Selective dual nuclear scanning in low-risk patients with chest pain to reliably identify and exclude acute coronary syndromes. Ann Emerg Med. 2001;38(3):207-15.

54. Schwitter J, Wacker CM, Wilke N, et al. MR-IMPACT II: Magnetic Resonance Imaging for Myocardial Perfusion Assessment in Coronary artery disease Trial: perfusion-cardiac magnetic resonance vs. single-photon emission computed tomography for the detection of coronary artery disease: a comparative multicentre, multivendor trial. Eur Heart J. 2012. 